\title{
Atorvastatin enhances apoptotic effects of tamoxifen on melanoma cancer cells
}

\author{
Ghasemi $\mathrm{M}^{1}$, Malek $\mathrm{M}^{1}$, Haghjooy Javanmard $\mathrm{Sh}^{1}$, Ghasemi $\mathrm{A}^{1}$, Naji Esfahani $\mathrm{H}^{1}$, Vaseghi $\mathrm{G}^{2}$ \\ Isfahan Cardiovascular Research Center, Cardiovascular Research Institute, Isfahan University \\ of Medical Sciences, Isfahan, Iran. golnazvaseghi@yahoo.com
}

\begin{abstract}
AIM: Tamoxifen engages mitochondrial estrogen receptor beta as an antagonist, increases mitochondrial cytotoxicity and induces tumor cell death. Tamoxifen also engages plasma membrane estrogen receptor alpha as an agonist, while it is suggested that in some users its activation is put into action by mechanism of resistance to tamoxifen. Apoptotic inducers have been shown to promote tamoxifen-induced cell death, which might be of great importance in overcoming tamoxifen resistance. Considering the pleiotropic effects of statins, in the present study, we investigated the effects of atorvastatin on tamoxifen-induced intrinsic apoptotic pathway activity in melanoma cells.

METHODS: Melanoma B16F10 cells were treated for 24 and $48 \mathrm{~h}$ with various concentrations of tamoxifen, atorvastatin and combination of tamoxifen + atorvastatin. Cells with no treatment were considered a control group, and the study was then followed by quantitative RT- PCR assay. Bax and cytochrome $c$ gene expressions were calculated by $\Delta \Delta$ ct method.

RESULTS: Co-treatment of atorvastatin + tamoxifen could strongly enhance the expression of pro/apoptotic factors of Bax and cytochrome $\mathrm{c}$ in melanoma cells compared to the tamoxifen and atorvastatin groups. CONCLUSION: In general, we conclude that the atorvastatin-induced increase in Bax and cytochrome c gene expression might be a permissive response to tamoxifen-induced cell death (Fig. 2, Ref. 37). Text in PDF www.elis.sk.

KEY WORDS: tamoxifen, atorvastatin, melanoma, Bax, cytochrome c.
\end{abstract}

\section{Introduction}

Melanoma is a cancer with high mortality risk. It originates in pigment-producing cells, i.e. melanocytes. The incidence rates are still growing globally and are highest among populations in environments with intense UV radiation (1). The advances in understanding the basic biology of melanoma in the past decade have yielded several new treatments. One such capacity involves the delineation of mitochondria modifications that occur during melanoma development, and effects of these changes on the response to therapy (2). BRAF-driven melanomas that have high mitochondrial contents exhibit increased oxidative phosphorylation and are more sensitive to mitochondria-targeted drugs (3). Tamoxifen, a well-known chemotherapeutic agent, engages mitochondrial estrogen receptor (ER) as an antagonist in human ma-

${ }^{1}$ Applied Physiology Research Center, Cardiovascular Research Institute, Department of Physiology, Isfahan University of Medical Sciences, Isfahan, Iran, and ${ }^{2}$ Isfahan Cardiovascular Research Center, Cardiovascular Research Institute, Isfahan University of Medical Sciences, Isfahan, Iran

Address for correspondence: G. Vaseghi, Dr, Cardiovascular Research Institute, Isfahan University of Medical Sciences, Isfahan, Iran. Phone: +98.313 .6692836$

Acknowledgements: This work was supported by the Isfahan University of Medical Sciences, Isfahan, Iran. lignant melanoma cells (4), and increases reactive oxygen species (ROS) concentrations from the mitochondria that are required for cytotoxicity. Induction of apoptosis via activation of c-Jun N-terminal kinase, p38 kinase and phosphorylation of c-Jun, expression of Fas ligand, up-regulation of BCL-2, Bax and caspases were observed in tamoxifen-treated cells $(5,6)$. When used with other apoptosis activators, tamoxifen was found to produce synergic effects, which might be of great importance in countering its or other drugs' resistance. For example, co-treatment with tamoxifen and TNF-related apoptosis-inducing ligand down-regulates antiapoptotic proteins, while simultaneously up-regulating the proapoptotic proteins regardless of their ER status (7). Researchers believe that the effects of this combination activate targets other than estrogen receptors on tumor cells that are key mediators of signaling pathways of cell survival or death and can reverse the resistance to chemotherapy. As a consequence, the consideration of new characteristics of tamoxifen is important in the treatment of solid tumors and particularly when used in combination with current target therapies $(5,8,9)$. In addition, the inhibition of mitochondrial oxidative phosphorylation has recently emerged as a promising strategy in the treatment of therapy-resistant cancer cells $(10,11)$. There is emerging evidence suggesting that statins, i.e. drugs well known for their hypocholesterolemic effects, impair mitochondria, which is demonstrable by abnormal mitochondrial morphology, decreased oxidative phosphorylation capacity, 
mitochondrial membrane potential reduction, generated ROS in mitochondria and activated intrinsic apoptotic pathway in carcinoma cell lines (12-14). As to the pleiotropic effects of statins, in the present study we were interested in understanding the effects of statins (atorvastatin) on tamoxifen-induced apoptotic cell death in melanoma cells.

\section{Materials and methods}

\section{Reagents}

Atorvastatin (calcium salt, (C33 H34FN2O5)2Ca 3H2O) and tamoxifen were obtained from Sobhan and Darupakhsh (Iran). Dulbecco's modified eagle medium (DMEM), fetal bovine serum (FBS), and trypsin were from Gibco-BRL (UK). The prepared stock solutions of compounds $(1 \mathrm{mM})$ in saline were diluted with the medium (DMEM) to reach 1,10 , and $100 \mu \mathrm{M}$ concentrations).

\section{Cell culture and treatments}

The B16F10 mouse melanoma cell line (obtained from Pasteur Institute) was cultured in DMEM supplemented with $10 \% \mathrm{FBS}$ at $37^{\circ} \mathrm{C}$ in a humidified atmosphere containing $5 \% \mathrm{CO}_{2}$. Cells were cultivated for 3-4 days to reach confluences of nearly $80 \%$. Then the melanoma cells were placed into two 96-well plates (1000 cells/well) and treated for 24 and 48 hours with varying doses of tamoxifen $(100,10$ and $1 \mu \mathrm{M})$, atorvastatin $(100,10$ and $1 \mu \mathrm{M})$ and co-treatment of tamoxifen and atorvastatin $(100,10,1 \mu \mathrm{M})$.

\section{Results}

The effect of tamoxifen and atorvastatin on Bax gene expression

The effects of tamoxifen, atorvastatin and their combination on Bax gene expression were evaluated in a melanoma cell line at different concentrations (final concentrations 1,10 , and100 $\mu \mathrm{M}$ ) after 24 and $48 \mathrm{~h}$ using RT- PCR assay (Fig. 1 A,B). As seen in Figure 1A, alternation of Bax gene expression was not observed after $24 \mathrm{~h}$ of incubation with drugs. Atorvastatin significantly promoted the expression of Bax gene at lower doses after $48 \mathrm{~h}$ of incubation (Fig. 1B) $(\mathrm{p}<0.05)$, while the rise of Bax gene expression was only observed in the trial with highest dose of tamoxifen. The data derived from the combined treatment showed the strongest result when lower doses of atorvastatin were combined with tamoxifen (any dose of tamoxifen). With an increase in the dose of atorvastatin, the toxicity effect was observed only in combination with high dose of tamoxifen $(100 \mu \mathrm{M})(\mathrm{p}<0.05)$.

The effect of tamoxifen and atorvastatin on cytochrome c gene expression

We examined whether atorvastatin can increase the expressions of cytochrome c gene in tamoxifen-treated melanoma cells. RTPCR analyses revealed (Fig. 2 B) that the administration of atorvastatin alone at high dose $(100 \mu \mathrm{M})(\mathrm{p}<0.05)$ could significantly increase the expression of cytochrome $\mathrm{c}$, while tamoxifen at low dose was observed to be effective after $48 \mathrm{~h}$ of incubation. The results of co-treatment showed that atorvastatin could, even at its ineffective dose markedly potentiate the concentration-dependent effect of tamoxifen on cytochrome $\mathrm{c}$ gene expression compared to control cells (DMEM-treated cells). The peak effect of co-treatment on cytochrome $\mathrm{c}$ expression was achieved in combination of atorvastatin at a dose of $10 \mu \mathrm{M}+$ tamoxifen at a dose of $100 \mu \mathrm{M}$ $(\mathrm{p}<0.001)$. With an increase in the dose of atorvastatin, the toxic effect was observed only in combination with tamoxifen at high doses. It is worthy to mention that cytochrome c gene expression was not changed after $24 \mathrm{~h}$ of drug co-treatment (Fig. $2 \mathrm{~A}$ ).
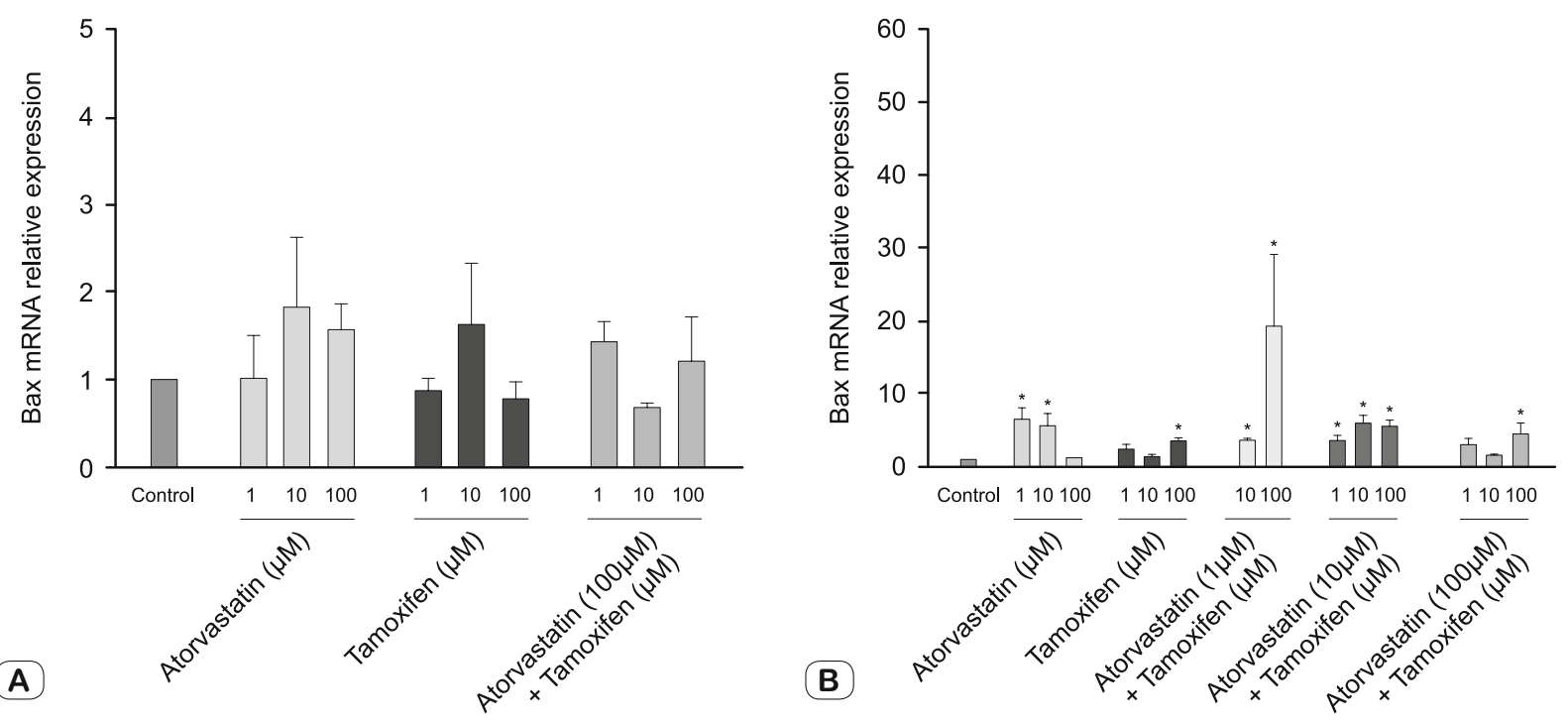

Fig. 1. Changes in Bax gene expression in melanoma cell line after incubation with different concentrations of tamoxifen, atorvastatin and their combination for 24 (A) and 48 (B) hours; * $p<0.05$ compared with the control. 

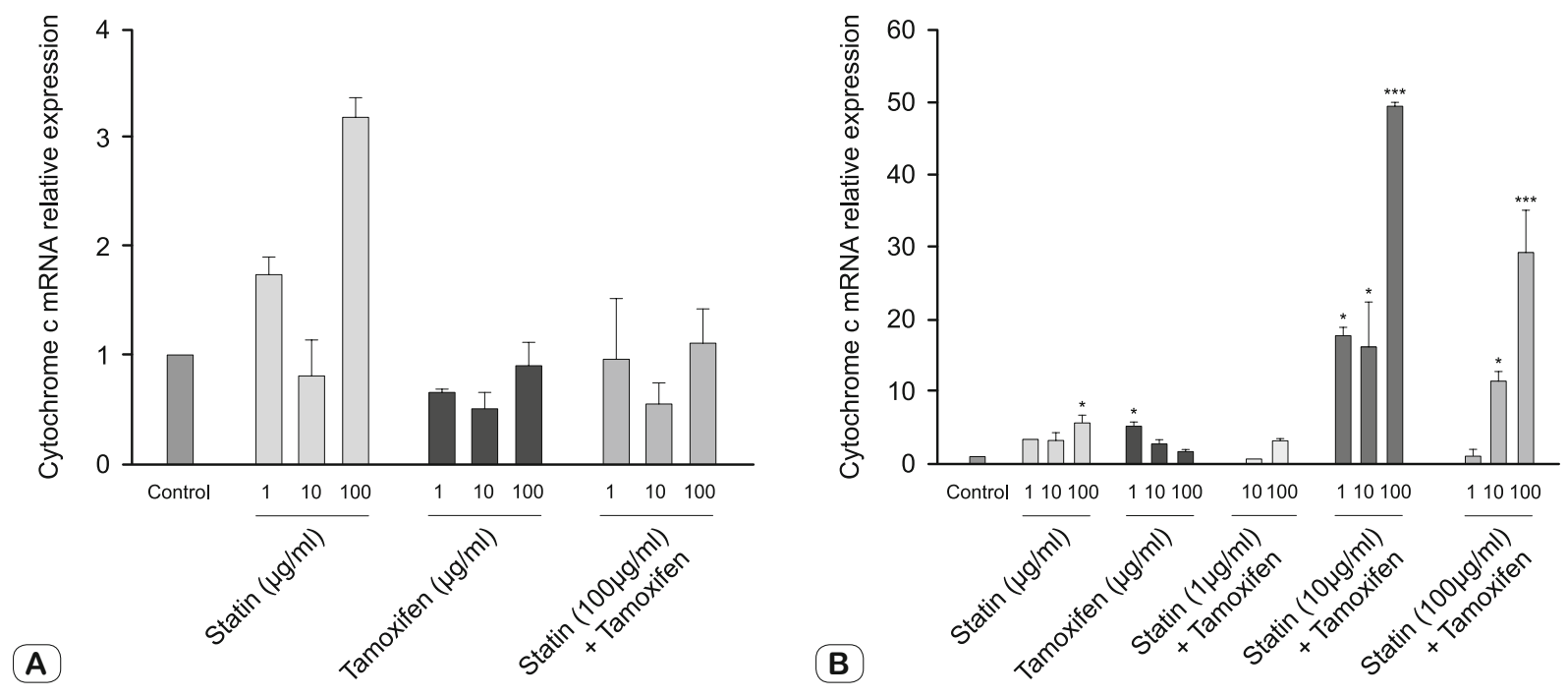

Fig. 2. Changes in cytochrome $\mathrm{c}$ gene expression in melanoma cell line after incubation with different concentrations of tamoxifen, atorvastatin and their combination for 24 (A) and 48 (B) hours; ${ }^{*} p<0.05, * * p<0.01$ and ${ }^{* * *} p<0.001$ compared with the control.

\section{Discussion}

Our previous data demonstrated that B16F10 mouse melanoma cells are responsive to toll-like receptor agonist and their responses are time- and dose-dependent (15). In this study, we investigated whether tamoxifen used in combination with d3-hydroxy-3-methylglutaryl coenzyme A reductase inhibitor (HRI), atorvastatin, can improve the tamoxifen's ability to enhance programmed cell death signaling. Our study showed a novel combination therapy regimen of atorvastatin + tamoxifen that augmented tamoxifen efficacy against melanoma cells in culture. Tamoxifen is the first and the oldest drug targeted on estrogen receptor (ER)-positive breast cancer (16). Recent experimental studies have revealed new biological effects of tamoxifen on tumor cells via targets other than estrogen receptors that are key mediators in tumor sensitivity to chemotherapy, particularly in the treatment of solid tumors $(4,5$, 17). The mitochondrial cell death is triggered by diverse cytotoxic stimuli including chemotherapeutic agents as well as by tamoxifen (18). These stimuli activate related BCL-2 family proteins (Bax/ BAK) leading to mitochondrial outer membrane permeabilization and thus to the release of apoptotic factor cytochrome c, and consequently, to recruitment and activation of apoptosis caspases $(5,6,19-21)$. BCL-2 family proteins are critical checkpoints of apoptotic cell death (22). Recent advances focus on direct targeting of Bax for cancer therapy (23). Cells lacking both Bax and BAK have proven to be completely apoptosis-resistant (24). The augmentation of tamoxifen apoptotic effects was observed when this antiestrogen was used in combination with apoptosis activators which might be of great importance in counteracting tamoxifen resistance. For instance, co-treatment with tamoxifen and TNF-related apoptosis-inducing ligand (TRAIL) down-regulates antiapoptotic proteins FLIP and BCL-2, while simultaneously up-regulating proapoptotic proteins FADD, tBid, Bax, caspases
8 and 9 in breast tumors, regardless of their ER status (7). Our study showed that part of the toxicity synergy of atorvastatin + tamoxifen is associated with the mitochondrial apoptosis pathway via increased proapoptotic Bax and apoptotic cytochrome $\mathrm{c}$ expression in melanoma cancer cells. First, we observed that the expression of pro-apoptotic proteins Bax was up-regulated moderately following B16F10 cells treatment just with tamoxifen while the peak of cytochrome c expression was achieved at its lowest dose $(1 \mu \mathrm{M})$ and decreased with an increase in tamoxifen concentrations. In addition, atorvastatin treatment alone led to a dose- and time-dependent increase in mentioned pro/apoptotic factors induction in melanoma cells. Finally, we observed that co-treatment of atorvastatin + tamoxifen could strongly synergize the expression of apoptotic factors in melanoma cells. Interestingly, in combined treatment, atorvastatin at a dose of $10 \mu \mathrm{M}$ had a more effective influence, approximately 50 -fold, and powerfully potentiated the toxicity at different doses of tamoxifen.

Statins are well known for their hypocholesterolemic effects. By inhibiting the metabolic-mevalonate pathway, they inhibit the activity of numerous GTPases of the RAS superfamily (25). Parallel studies of different cancer cells demonstrated that statins mediate its cytotoxic effects mostly via the intrinsic apoptotic pathway. Simvastatin, fluvastatin and atorvastatin in a time- and dose-dependent manner induce pro-apoptotic BCL2 family members, Bax and ROS in breast cancer cell lines (26-31). Shellman et al. have also pointed to the fact that in mice and human models of melanoma, lovastatin enhances activity of apoptotic factor caspase-3 up to 50-fold (32). Atorvastatin has been shown to induce apoptosis in activated hepatic stellate cells and to increase highly the protease activity of caspase- 9 and caspase-3 (33). Our data also showed that atorvastatin treatment alone led to a dose- and time-dependent increase by $8.5 \%$ in the induction of Bax and cytochrome $\mathrm{c}$ in melanoma cells. Now, one question remains, namely 
how atorvastatin as a mevalonate pathway inhibitor opens the way for tamoxifen to induce a powerful apoptotic response. Based on studies, we propose some mechanisms of cell function that can mediate this beneficial statin effect, namely downregulation of survivin expression (34), and increase in reactive oxygen species (ROS) and nitric oxide synthase activity (iNOS or NOS II) (28, 35). Previously, it has been demonstrated that statins effectively down-regulate the expression of survivin protein in colon cancer and MCF-7 cells (34). These results suggest that survivin acts as a factor inducing resistance against tamoxifen-induced apoptosis, and the combined use of tamoxifen and statin enhances the toxic behavior of tamoxifen in breast cancer cells, which may be a novel approach to raising the tamoxifen toxicity in other cancers. Tamoxifen engages the mitochondrial estrogen receptor beta $(E R \beta)$ as an antagonist and increases reactive oxygen species (ROS) concentrations from the mitochondria in part through down-regulating manganese superoxide dismutase (MnSOD) activity that is required for cytotoxicity (36). ROS activates protein kinase $\mathrm{C}$ delta and c-jun $\mathrm{N}$-terminal kinases, resulting in the mitochondrial translocation of Bax and cytochrome c release (23). Interestingly in some studies, tamoxifen failed to cause high ROS levels due to the stimulation of MnSOD activity through agonistic effects at mitochondrial $\operatorname{ER} \beta(36)$. We also observed an apparently low concentration of tamoxifen antagonized ER-dependent pathways and increased Bax expression, while high concentration of tamoxifen acted through its agonistic effects. Some studies have indicated that low concentrations of tamoxifen activate ER-dependent pathways, while high concentrations of tamoxifen induce the oxidative stress reaction and activate non-ER pathways to induce cell apoptosis $(5,37)$. It seems that melanoma MnSOD can be therapeutically targeted with atorvastatin (or statins) to switch tamoxifen effects (or reverse tamoxifen resistance) and enhance the apoptosis response. However, more pharmacological studies are required for determining the role of MnSOD in co-treatment effects of statins and tamoxifen in cancers.

In general, we conclude that the atorvastatin-induced increase in Bax and cytochrome $\mathrm{c}$ gene expressions might be a permissive response to tamoxifen-induced cell death.

\section{References}

1. Ali Z, Yousaf N, Larkin J. Melanoma epidemiology, biology and prognosis. EJC Suppl 2013; 11 (2): 81-91. doi: 10.1016/j.ejcsup. 2013.07.012.

2. Theodosakis N, Micevic G, Kelly DP, Bosenberg M. Mitochondrial function in melanoma. Archives of biochemistry and biophysics 2014; 563: 56-59. doi: 10.1016/j.abb.2014.06.028.

3. Weinberg SE, Chandel NS. Targeting mitochondria metabolism for cancer therapy. Nature Chem Biol 2015; 11 (1): 9-15. doi: 10.1038/nchembio. 1712 .

4. Matsuoka H, Tsubaki M, Yamazoe Y, Ogaki M, Satou T, Itoh T et al. Tamoxifen inhibits tumor cell invasion and metastasis in mouse melanoma through suppression of $\mathrm{pkc} / \mathrm{mek} / \mathrm{erk}$ and $\mathrm{pkc} / \mathrm{pi} 3 \mathrm{k} / \mathrm{akt}$ pathways. Exp Cell Res 2009; 315 (12): 2022-2032. doi: 10.1016/j.yexcr.2009.04.009.
5. Bogush T, Dudko E, Bogush E, Polotsky B, Tjulandin S, Davydov M. Tamoxifen non-estrogen receptor mediated molecular targets. Oncol Rev 2012; 6 (2). e15. doi: 10.4081/oncol.2012.e15.

6. Han P, Kang JH, Li HL, Hu SX, Lian HH, Qiu PP et al. Antiproliferation and apoptosis induced by tamoxifen in human bile duct carcinoma qbc939 cells via upregulated p53 expression. Biochem Biophys Res Comm 2009; 385 (2): 251-256. doi: 10.1016/j.bbrc.2009.05.059.

7. Lagadec C, Adriaenssens E, Toillon R, Chopin V, Romon R, Van Coppenolle $\mathbf{F}$ et al. Tamoxifen and trail synergistically induce apoptosis in breast cancer cells. Oncogene 2008; 27 (10): 1472-1477.

8. Zulehner N, Maurer M, Węsierska-Gądek J. Effect of anti-estrogen combined with roscovitine, a selective cdk inhibitor, on human breast cancer cells differing in expression of er. J Exp Ther Oncol 2011; 9 (1): 17-25.

9. Velho TR. Metastatic melanoma - a review of current and future drugs. Drugs in context 2012; 2012: 212242. doi: 10.7573/dic.212242 .

10. Kuntz EM, Baquero P, Michie AM, Dunn K, Tardito S, Holyoake TL et al. Targeting mitochondrial oxidative phosphorylation eradicates therapy-resistant chronic myeloid leukemia stem cells. Nature Med 2017; 23 (10): 1234. doi: 10.1038/nm.4399.

11. Corazao-Rozas P, Guerreschi P, André F, Gabert P-E, Lancel S, Dekiouk $\mathrm{S}$ et al. Mitochondrial oxidative phosphorylation controls cancer cell's life and death decisions upon exposure to mapk inhibitors. Oncotarget 2016; 7 (26): 39473. doi: 10.18632/oncotarget. 7790.

12. Cafforio P, Dammacco F, Gernone A, Silvestris F. Statins activate the mitochondrial pathway of apoptosis in human lymphoblasts and myeloma cells. Carcinogenesis 2005; 26 (5): 883-891. doi: 10.1093/carcin/bgi036.

13. Spampanato C, De Maria S, Sarnataro M, Giordano E, Zanfardino M, Baiano $\mathbf{S}$ et al. Simvastatin inhibits cancer cell growth by inducing apoptosis correlated to activation of Bax and down-regulation of bcl-2 gene expression. International journal of oncology 2012; 40 (4): 935-941. doi: $10.3892 /$ ijo.2011.1273.

14. Blanco-Colio LM, Villa A, Ortego M, Hernández-Presa MA, Pascual A, Plaza JJ et al. 3-hydroxy-3-methyl-glutaryl coenzyme a reductase inhibitors, atorvastatin and simvastatin, induce apoptosis of vascular smooth muscle cells by downregulation of bcl-2 expression and rho a prenylation. Atherosclerosis 2002; 161 (1): 17-26. doi: doi.org/10.1016/ S0021-9150 (01)00613-X.

15. Dana N, Javanmard SH, Vaseghi G. Effect of lipopolysaccharide on toll-like receptor-4 signals in mouse cancer cells. Bratisl Lek Listy 2017; 118 (10): 598-601. doi: 10.4149/BLL_2017_115.

16. Jordan VC. Tamoxifen: Catalyst for the change to targeted therapy. Eur J Cancer 2008; 44 (1): 30-38. doi: 10.1016/j.ejca.2007.11.002.

17. Han P, Kang JH, Li HL, Hu SX, Lian HH, Qiu PP et al. Antiproliferation and apoptosis induced by tamoxifen in human bile duct carcinoma qbc939 cells via upregulated p53 expression. Biochem Biophys Res Commun 2009; 385 (2): 251-256. doi: 10.1016/j.bbrc.2009.05.059.

18. Nazarewicz RR, Zenebe WJ, Parihar A, Larson SK, Alidema E, Choi J et al. Tamoxifen induces oxidative stress and mitochondrial apoptosis via stimulating mitochondrial nitric oxide synthase. Cancer Res 2007; 67 (3): 1282-1290. doi: 10.1158/0008-5472.CAN-06-3099.

19. Lavie Y, Zhang Zc, Cao Ht, Han TY, Jones RC, Liu YY et al. Tamoxifen induces selective membrane association of protein kinase c epsilon in mcf-7 human breast cancer cells. International journal of cancer 1998; 77 (6): 928-932. doi: doi.org/10.1002/ (SICI)1097-0215 (19980911)77:6<928::AID-IJC22>3.0.CO; 2-W. 


\section{$752-756$}

20. Thiantanawat A, Long BJ, Brodie AM. Signaling pathways of apoptosis activated by aromatase inhibitors and antiestrogens. Cancer Res 2003; 63 (22): 8037-8050.

21. Jürgensmeier JM, Xie Z, Deveraux Q, Ellerby L, Bredesen D, Reed JC. Bax directly induces release of cytochrome $\mathrm{c}$ from isolated mitochondria. Proc Nat Acad Sci 1998; 95 (9): 4997-5002.

22. Danial NN. Bcl-2 family proteins: Critical checkpoints of apoptotic cell death. Clin Cancer Res 2007; 13 (24): 7254-7263. doi: 10.1158/10780432.CCR-07-1598.

23. Liu Z, Ding Y, Ye N, Wild C, Chen H, Zhou J. Direct activation of Bax protein for cancer therapy. Med Res Rev 2016; 36 (2): 313-341. doi: 10.1002/med.21379.

24. Wei MC, Zong W-X, Cheng EH-Y, Lindsten T, Panoutsakopoulou V, Ross AJ et al. Proapoptotic Bax and bak: A requisite gateway to mitochondrial dysfunction and death. Science 2001; 292 (5517): 727-730. doi: $10.1126 /$ science. 1059108 .

25. Alizadeh J, Zeki AA, Mirzaei N, Tewary S, Moghadam AR, Glogowska A et al. Mevalonate cascade inhibition by simvastatin induces the intrinsic apoptosis pathway via depletion of isoprenoids in tumor cells. Sci Reports 2017; 7: 44841. doi: 10.1038/srep44841.

26. Shen Y, Du Y, Zhang Y, Pan Y. Synergistic effects of combined treatment with simvastatin and exemestane on mcf-7 human breast cancer cells. Mol Med Report 2015; 12 (1): 456-462. doi: 10.3892/mmr. 2015.3406

27. Shen YY, Yuan Y, Du YY, Pan YY. Molecular mechanism underlying the anticancer effect of simvastatin on MDA-MB-231 human breast cancer cells. Mol Med Reports 2015; 12 (1): 623-630. doi: 10.3892/ mmr.2015.3411.

28. Sánchez CA, Rodríguez E, Varela E, Zapata E, Paez A, Massó FA et al. Statin-induced inhibition of mcf-7 breast cancer cell proliferation is related to cell cycle arrest and apoptotic and necrotic cell death mediated by an enhanced oxidative stress. Cancer Invest 2008; 26 (7): 698-707. doi: $10.1080 / 07357900701874658$.
29. Kwok SC, Samuel SP, Handal J. Atorvastatin activates heme oxygenase-1 at the stress response elements. J Cell Mol Med 2012; 16 (2): 394-400. doi: 10.1111/j.1582-4934.2011.01324.x.

30. Hwang KE, Kim YS, Hwang YR, Kwon SJ, Park DS, Cha BK et al. Enhanced apoptosis by pemetrexed and simvastatin in malignant mesothelioma and lung cancer cells by reactive oxygen species-dependent mitochondrial dysfunction and bim induction. Internat J Oncol2014; 45 (4): 1769-1777. doi: 10.3892/ijo.2014.2584.

31. Hwang KE, Park C, Kwon SJ, Kim YS, Park DS, Lee MK et al. Synergistic induction of apoptosis by sulindac and simvastatin in a549 human lung cancer cells via reactive oxygen species-dependent mitochondrial dysfunction. Internat J Oncol 2013; 43 (1): 262-270. doi: 10.3892/ijo.2013.1933.

32. Shellman YG, Ribble D, Miller L, Gendall J, VanBuskirk K, Kelly D et al. Lovastatin-induced apoptosis in human melanoma cell lines. Melanoma Res 2005; 15 (2): 83-89.

33. Aprigliano I, Dudas J, Ramadori G, Saile B. Atorvastatin induces apoptosis by a caspase-9-dependent pathway: An in vitro study on activated rat hepatic stellate cells. Liver International 2008; 28 (4): 546-557. doi: 10.1111/j.1478-3231.2008.01682.x.

34. Moriai R, Tsuji N, Moriai M, Kobayashi D, Watanabe N. Survivin plays as a resistant factor against tamoxifen-induced apoptosis in human breast cancer cells. Breast Cancer Res Treat 2009; 117 (2): 261-271. doi: 10.1007/s10549-008-0164-5.

35. Kotamraju S, Willams CL, Kalyanaraman B. Statin-induced breast cancer cell death: Role of inducible nitric oxide and arginase-dependent pathways. Cancer Res 2007; 67 (15): 7386-7394. doi: 10.1158/0008-5472.

36. Razandi M, Pedram A, Jordan VC, Fuqua S, Levin ER. Tamoxifen regulates cell fate through mitochondrial estrogen receptor beta in breast cancer. Oncogene 2013; 32 (27): 3274. doi: 10.1038/onc.2012.335.

37. Obrero M, David VY, Shapiro DJ. Estrogen receptor-dependent and estrogen receptor-independent pathways for tamoxifen and 4-hydroxytamoxifen-induced programmed cell death. Journal of Biological Chemistry 2002; 277 (47): 45695-45703. doi: 10.1074/jbc.M208092200. 